\title{
Validación en trabajadores peruanos de la versión ultra reducida del Utrech Work Engagement
}

\author{
Ultra short version of Utrech Work Engagement Scale: \\ validation in Peruvian workers
}

\footnotetext{
Gustavo Alexis Calderón-De la Cruz ${ }^{1}$ (D) 0000-0001-8780-7517

César Merino-Soto ${ }^{2}$ (i) 0000-0002-1407-8306

Ioanna Francesca Reyes-Ayala ${ }^{3}$ (D) 0000-0001-9123-4600

Katherin Victoria Luna-Yon ${ }^{3}$ (D) 0000-0003-4752-4154
Universidad de San Martín de Porres, Facultad de Ciencias de la Comunicación, Turismo y Psicología, Instituto de Investigación de la Escuela de Psicología, Lima, Perú.

¿Universidad Autónoma del Estado de Morelos, Cuernavaca, México.

Universidad de San Martín de Porres, Facultad de Ciencias de la Comunicación, Turismo y Psicología, Lima, Perú.
}

Fechas · Dates

Recibido: 28/02/2021

Aceptado: 13/01/2022

Publicado: 17/01/2022
Correspondencia · Corresponding Author

Gustavo Alexis Calderón De la Cruz

Universidad de San Martín de Porres, Lima, Perú

Email: gcalderond@usmp.pe 


\title{
Resumen
}

Evaluar los efectos positivos de los factores psicosociales del trabajo es una permanente iniciativa de investigadores y profesionales. El objetivo del estudio es examinar psicométricamente la versión ultra reducida del Utrech Work Engagement Scale (UWES-3). Participaron 200 trabajadores de 21 a 53 años procedentes de la ciudad de Lima (Perú). Las evidencias de validez corroboradas fueron su estructura interna y su relación con otros constructos; los coeficientes alfa de Cronbach (a), Omega ( $\omega$ ) y H fueron los estimadores para la confiabilidad. Como resultados, se demostró la unidimensionalidad del UWES-3 y su asociación negativa con el síndrome de burnout; los niveles de confiabilidad en los tres estimadores fueron altos. Los resultados iniciales de validez y confiabilidad del UWES-3 pueden considerarse satisfactorios e incentivan a la continuidad de su estudio en el trabajo peruano.

Palabras clave: Estudio de validación; Psicometría; Compromiso laboral; Agotamiento profesional; Perú.

\begin{abstract}
Researchers and practicing professionals are continually assessing the positive effects of psychosocial factors at work. This study psychometrically examined use of the ultra-short version of the Utrech Work Engagement Scale (UWES-3). Two hundred workers in Lima, Peru participated, ranging from 21 to 53 years of age. We corroborated the validity of the scale, according to its internal structure and relationship with other constructs; Cronbach alpha (a), omega $(\omega)$ and $\mathrm{H}$ coefficients were the reliability estimators. Results confirmed the unidimensionality of the UWES-3 and its negative association with burnout syndrome; scale reliability, based on the three estimators, was high. The initial validity and reliability results of the UWES-3 can be considered satisfactory and encourage its continued use in the Peruvian workforce.
\end{abstract}

Keywords: Validation study; Psychometrics; Work Engagement; Burnout, Professional; Peru. 


\section{Introducción}

El work engagement es un estado afectivo-cognitivo, positivo, que es persistente en el tiempo y está asociado a experiencias vivenciadas en el trabajo(1). El vigor (energía, resistencia mental, voluntad y persistencia frente a las dificultades del trabajo), la dedicación (fuerte involucración e identificación con el trabajo) y la absorción (concentración, disfrute y dificultad para desvincularse del trabajo), son componentes que explican su desarrollo(2).

Los estudios iniciales sobre el engagement aparecieron para evidenciar su relación opuesta con el síndrome de burnout, no obstante, tomando el modelo Demandas-Recursos Laborales ${ }^{(3)}$, el engagement principalmente depende de un proceso motivacional, incrementándose en presencia de los recursos laborales (por ejemplo el apoyo social), las demandas laborales desafiantes y los recursos personales (por ejemplo autoeficacia). Se sostiene que trabajadores con engaged mejoran en su desempeño y compromiso ${ }^{(4,5)}$.

El Utrech Work Engagement Scale (UWES) es la medición más utilizada del engagement. La escala originalmente presentó 17 ítem (UWES-17)(1) integrando las dimensiones de vigor, dedicación y absorción; posteriormente, una versión breve aparecería con 9 ítems (UWES-9)(6). Las propiedades psicométricas en estas dos versiones fueron aceptables en sus contextos de desarrollo. No obstante, una revisión en 21 estudios demostró la existencia de ambigüedades en la estructura factorial del UWES(7). Esta situación supone un reto en los estudios psicométricos con la escala.

Actualmente existe una versión ultra reducida de 3 ítems (UWES-3) validada en 5 países, entre ellos España ${ }^{(2)}$. Un ítem fue seleccionado de cada dimensión mediante un proceso de validez aparente. Mediante un análisis factorial confirmatorio, el UWES-3 discriminó mediciones relacionadas con el malestar en el trabajo (como el síndrome de burnout). Así mismo, el UWES-3 se asoció con el UWES-9 y demostró relaciones teóricamente esperables con constructos integrados en el modelo Demandas-Recursos Laborales. Los resultados obtenidos corroboraron la utilidad de esta versión ultra reducida para la medición del engagement.

Este avance alentador sobre las propiedades psicométricas del UWES-3 no ha sido replicado en contextos que incluyan participantes de habla hispana de otros países, como es el caso de Perú, donde únicamente existe un estudio de validación con el UWES original(8). Por lo tanto, se necesitan estudios que verifiquen su validez y que faciliten la continuidad de su uso e interpretación. Además, dado que la evaluación de los factores psicosociales requiere de altos costes de tiempo, esfuerzo y reacciones emocionales negativas de los evaluados, una escala tan breve aporta a la reducción de estos problemas siendo de beneficios para las organizaciones.

Por lo tanto, el objetivo del estudio es verificar las propiedades psicométricas del UWES-3 en trabajadores peruanos, al examinar la validez de su estructura interna y su relación con otros constructos; considerando en este último caso la asocia- 
ción negativa del engagement con el síndrome de burnout según resultados de estudios previos ${ }^{(1,2)}$.

\section{Métodos}

\section{Participantes}

Los criterios de inclusión fueron los siguientes: 1) trabajadores en el rubro (sector) de belleza, 2) peruanos, 3) mayores de 18 años, 4) que cumplieran un contrato de más de 6 meses, y 5) experiencia en el puesto superior a un año. El muestreo fue no probabilístico de tipo intencional. Finalmente, la muestra estuvo formada por 200 trabajadores.

\section{Instrumentos}

Utrech Work Engagement Scale-3(1), que integra los ítems: 1 (Vigor: Vi), 8 (Dedicación: De) y 14 (Absorción: Abs)(8). Con opciones de respuesta escaladas ordinalmente de 0 (Nunca-Ninguna vez) a 6 (Siempre-Todos los días).

Maslach Burnout Inventory-General Survey (MBI-GS)(9), que contiene las dimensiones de Agotamiento Emocional (AE: 5 ítems), Indiferencia (Ind: 5 ítems) y Eficacia Profesional (EP: 6 ítems). Con opciones de respuesta escaladas ordinalmente de 0 (Nunca-Ninguna vez) a 6 (Siempre-Diariamente). Se utilizó la versión peruana, en que se eliminó el ítem 13 (Indiferencia), quedando una propuesta de 15 ítems ${ }^{(10)}$.

\section{Procedimiento}

El estudio se llevó a cabo en la ciudad de Lima entre el año 2018 y 2019. Tras ser aceptado el proyecto por los directivos de la organización, se programó la evaluación que consistió en rellenar una ficha sociodemográfica y los dos cuestionarios. Durante la evaluación se siguieron los principios de la Declaración de Helsinski, garantizando una participación voluntaria, el anonimato, la confidencialidad y la inocuidad. Todos los participantes firmaron un consentimiento informado.

\section{Análisis de datos}

Después de verificar que las encuestas se habían completado adecuadamente, se realizaró el análisis de los datos usando el programa R studio. Inicialmente, se examinó la presencia de los outliers multivariados en el UWES-3 usando el estadístico de la distancia de Mahalanovis $\left(D^{2}\right)$. Como resultado, se conservó la totalidad de la muestra.

Los estadísticos descriptivos fueron: Media (M), Desviación Típica (DT), asimetría (g1) y curtosis (g2). Para la estructura interna se aplicó un análisis factorial confirmatorio (modelamiento de ecuaciones estructurales) y se estimó el coeficiente ra- 
tio Sign-to-Noise (STNR) para cada ítem, basado en la replicabilidad del constructo obtenido mediante el coeficiente $\mathrm{H}^{(11)}$.

Dos modelos fueron evaluados: a) de medidas independientes en el UWES-3 y el MBI-GS y, b) adhiriendo la dimensión de EP (MBI-GS) al UWES-3. El estimador fue de mínimos cuadrados ponderados para variables categóricas (WLSMV- $\chi^{2}$ ) y el ajuste se definió con los índices más robustos y frecuentes considerando los siguientes niveles: $>.95$ (bueno) en CFI y TLI y<.05 para el RMSEA(12).

La confiabilidad fue obtenida con los coeficientes omega $(\omega)^{(13)}$, alfa $(a)^{(14)}$, el coeficiente de reproducibilidad del constructo $(H)^{(11)}$, esperando en todos niveles $>.70$. Los intervalos de confianza fueron obtenidos con simulación Bootstrap ${ }^{(15)}$.

\section{Resultados}

Características de la muestra. El 60\% fueron mujeres entre 21 y 53 años ( $M=$ 33.32; DT $=4.27$ ) provenientes de la ciudad de Lima. Los puestos de trabajo fueron de consultor de belleza (59\%), maquilladoras (31\%), gerente de ventas al por menor (8\%) y entrenadores personales (0.2\%). Entre 1 y 9 años $(M=6.43$; DT = 2.44) fue la experiencia laboral, contando con contratos de tipo definido (72\%) y temporal (28\%).

Descriptivos. Los ítems del MBI-GS y UWES-3 mantuvieron normalidad estadística siendo los ítems 9 y 14 (dimensión de Ind) los de mayor exceso de curtosis en sus distribuciones. La tendencia de respuesta fue similar en las dimensiones AE e Ind (opciones entre 0 y 1), mientras que la EP y los ítems del UWES-3 tuvieron mayor densidad de respuesta alrededor de las opciones 3 y 4 (Tabla 1).

Estructura interna. En la Tabla 1 aparecen los parámetros para los ítems del UWES-3 y MBI-GS, así como sus relaciones latentes. El modelamiento en conjunto entre el MBI-GS y UWES-3 produjo un buen ajuste WLSMV- $\chi^{2}=56.51, \mathrm{gl}=$ $129, p<.01 ; \mathrm{CFI}=.981 ; \mathrm{TLI}=.980 ; \mathrm{RMSEA}=.053(\mathrm{IC90 \%}=.024, .075)$. Las cargas factoriales en el UWES-3 y MBI-GS fueron predominantemente altas (>.650). No se detectaron errores correlacionados significativos. El coeficiente STNR puso en relevancia la diferencia de varianza retenida en cada ítem del UWES-3.

Relación con otros constructos. La correlación latente UWES-3 - MBI-GS (última sección de la Tabla 1), mostró alta fuerza asociativa y dirección negativa con AE e Ind, y muy alta covariación positiva entre el UWES-3 y EP. Para evaluar más de cerca la diferenciación entre ambas variables latentes, se modeló nuevamente el UWES-3 con el MBI-GS, pero especificando los ítems de engagement dentro de EP. Aunque el ajuste fue satisfactorio en términos absolutos y con cargas factoriales aceptables ( $\lambda$ entre .434 y .648) este fue inferior al modelo inicial: WLSMV- $\chi^{2}=$ 186.26, $\mathrm{gl}=132, \mathrm{p}<.01 ; \mathrm{CFI}=.972 ; \mathrm{TLI}=.970 ; \mathrm{RMSEA}=.064(\mathrm{IC} 90 \%=.041, .084)$, sugiriendo que la variabilidad de respuesta en los ítems del UWES-3 no es suficientemente grande respecto a EP comparada con el propio factor del UWES-3. La homogeneidad de los ítems ${ }^{(16)}$ en UWES-3 fue satisfactoria, robusto- $F=.0, p=1.0$. 
Tabla 1: Parámetros de los ítems y confiabilidad del UWES-3 y el MBI-GS

\begin{tabular}{|c|c|c|c|c|c|c|c|c|c|}
\hline & \multicolumn{4}{|c|}{ Estadísticos descriptivos } & \multicolumn{5}{|c|}{ Análisis Factorial Confirmatorio } \\
\hline & M & DT & g1 & g2 & $\begin{array}{c}\mathrm{AE} \\
\lambda\end{array}$ & $\begin{array}{c}\mathrm{EP} \\
\lambda\end{array}$ & $\begin{array}{c}\text { Ind } \\
\lambda\end{array}$ & $\begin{array}{c}\text { UWES-3 } \\
\lambda\end{array}$ & STNR \\
\hline \multicolumn{10}{|l|}{ MBI-GS } \\
\hline AE1 & 1.49 & .904 & .615 & 1.589 & .677 & & & & .934 \\
\hline AE2 & 1.61 & .886 & .502 & .673 & .573 & & & & .518 \\
\hline AE3 & 1.27 & .908 & 1.331 & 1.950 & .683 & & & & .855 \\
\hline AE4 & 1.12 & .795 & .642 & .980 & .786 & & & & 1.429 \\
\hline AE6 & .90 & 847 & .703 & .418 & .709 & & & & 1.028 \\
\hline EP5 & 3.90 & 1.314 & -.684 & .466 & & .636 & & & .672 \\
\hline EP7 & 3.93 & 1.174 & -.129 & -.952 & & .734 & & & 1.203 \\
\hline EP10 & 4.11 & 1.230 & -.778 & .948 & & .751 & & & 1.089 \\
\hline EP11 & 4.10 & 1.159 & -.953 & 1.638 & & .789 & & & 1.466 \\
\hline EP12 & 3.96 & 1.188 & -.842 & .956 & & .809 & & & 1.950 \\
\hline EP16 & 3.86 & 1.271 & -.875 & 1.036 & & .703 & & & 1.182 \\
\hline Ind8 & 1.00 & .791 & .749 & .574 & & & .774 & & 1.318 \\
\hline Ind9 & 1.14 & .921 & 1.218 & 2.809 & & & .649 & & .638 \\
\hline Ind14 & 1.04 & 1.034 & 2.380 & 8.101 & & & .617 & & .728 \\
\hline Ind15 & .93 & .756 & .691 & .577 & & & .611 & & .683 \\
\hline \multicolumn{10}{|l|}{ UWES-3 } \\
\hline Vi1 & 3.48 & 1.168 & .107 & -.770 & & & & .784 & 1.595 \\
\hline De8 & 3.56 & 1.183 & .097 & -.617 & & & & .871 & 3.143 \\
\hline Abs14 & 3.05 & .097 & .327 & .180 & & & & .670 & 815 \\
\hline \multicolumn{10}{|c|}{ Confiabilidad } \\
\hline$a$ & & & & & .838 & .927 & .650 & .825 & \\
\hline$\omega$ & & & & & .817 & .878 & .759 & .821 & \\
\hline $\mathrm{H}$ & & & & & .829 & .885 & .774 & .847 & \\
\hline \multicolumn{10}{|c|}{ Correlaciones } \\
\hline$A E$ & & & & & 1 & & & & \\
\hline EP & & & & & $-.523 \star \star$ & 1 & & & \\
\hline Ind & & & & & $.835^{\star \star}$ & $-.474 \star \star$ & 1 & & \\
\hline UWES-3 & & & & & $-.420 \star \star$ & $.791^{\star \star}$ & $-.412^{\star \star}$ & & \\
\hline $\begin{array}{l}: \text { Cargas fa } \\
\star \star \\
\text { a: coeficien } \\
\omega: 01 \\
\omega: \text { coeficier } \\
H: \text { coeficient }\end{array}$ & ffiabilic & $\begin{array}{l}\text { d alfa de } \\
\text { ad Omeg } \\
\text { del cons }\end{array}$ & Cronbach & & & & & & \\
\hline
\end{tabular}


Confiabilidad. La confiabilidad y la replicabilidad de los constructos medidos por el UWES-3 es elevada (>.80); poblacionalmente, también se muestran valores aceptables para el coeficiente $\mathrm{W}$ (boostrap IC95\%: .730, .881, error estándar = .036) y para el coeficiente $a$ (bootstrap IC95\%: .731, .881, error estándar $=.037$ ). La diferencia entre los coeficientes a y w fueron inconsecuentes $(<|.06|)^{(17)}$, y la característica de tau-equivalencia de los ítems (robusto- $F=.819, p>.30)^{(18)}$ puede considerarse satisfecha. En función de la variabilidad (DT = 2.99) y la confiabilidad a de los puntajes, el error estándar de medición (1.251) en el 95\% de confianza fue 2.452 .

La información descriptiva adicional de los puntajes consiste en una $M=10.09$, $\mathrm{g} 1=.210$ (error estándar $=.241)$ y g2 $=-.465$ (error estándar $=.478$ ), sugiriendo un desvío no significativo de la normalidad estadística (D'Agostino-Pearson $K^{2}=$ $1.975, p>$.30).

\section{Discusión}

Los resultados de validez del UWES-3, examinando su unidimensionalidad a partir del análisis de su estructura interna y su relación negativa con el burnout, puede considerarse satisfactoria respaldando la propuesta del estudio original(2).

Un aspecto para considerar fue la asociación cercana del UWES-3 con la EP (dimensión del MBI-GS). Instrumentalmente, la ineficacia profesional (proceso del burnout) al ser evaluada con ítems positivos (EP) no presenta fuertemente asociación con las dimensiones del burnout, por el contrario, tiene mayor relación con el engagement ${ }^{(19)}$. Esto es convergente con el modelo Demandas-Recursos Laborales, donde se sostiene que la EP es un recurso laboral orientado a explicar el proceso del engagement.(4). En ese sentido, la asociación encontrada entre la EP y el engagement, no puede desafiar la validez del estudio porque es razonable que la covariación entre ambas variables sea elevada.

Por otro lado, la consistencia interna fue elevada (> .80), y para una escala con solo 3 ítems, esta magnitud puede ser óptima y replicable debido a la fuerza de la varianza retenida, y comparada con la tendencia del estudio original. Nuestros resultados también sugieren el uso indiferenciado del coeficiente $\omega$ y a para representar la varianza de error en el puntaje, un aspecto que fue acompaño de la homogeneidad y similar validez de los ítems; este resultado sirve como precedente para tratar la descripción de los participantes.

Entre las limitaciones del estudio, se menciona la relativa al pequeño tamaño muestral y la homogeneidad laboral. Como recomendaciones sugeridas para futuros estudios, se espera replicar el análisis de la validez en diversos contextos ocupacionales, estudiar su comparativa con el UWES-9, la invarianza de medición y evaluar el valor predictivo del UWES-3 desde su papel de variable endógena (conociendo el efecto de las demandas y los recursos laborales y los recursos personales) o exógena (analizando su efecto con variables organizacionales y personales de salida). 
Como conclusión, el estudio respalda la potencial utilidad del UWES-3 para la medición del engagement, siendo aplicable en evaluaciones extensivas que estén orientas en explorar los factores psicológicos que emergen de las condiciones del trabajo.

\section{Agradecimientos}

Se agradece la participación de quienes estuvieron involucrados en el estudio.

\section{Financiación}

Este trabajo no cuenta con financiación institucional.

\section{Conflicto de intereses}

No existe conflicto de intereses de los autores del estudio.

\section{Bibliografía}

1. Schaufeli WB, Salanova M, Gonzáles-Romá V, Bakker AB. The measurement of engagement and burnout: a two sample confirmatory factor analytic approach. J Happiness Stud. 2002;3(1):71-92. doi: 10.1023/A:1015630930326

2. Schaufeli WB, Shimazu A, Hakanen J, Salanova M, De Witte H. (2017). An ultra-short measure for work engagement: the UWES-3 validation across five countries. Eur J Psychol Assess. 2019;35(4):577-591. doi: 10.1027/1015-5759/a000430

3. Lesener T, Gusy B, Wolter C. The job demands-resources model: A meta-analytic review of longitudinal studies. Work \& Stress. 2018;33(1):1-28. doi:10.1080/0267 8373.2018.1529065

4. Bakker AB, Demerouti E. Job Demands-Resources Theory. In: Chen PY, Cooper $\mathrm{CL}$, Eds. Work and Wellbeing: A complete Reference Guide. New York: John Wiley \& Sons; 2014. p. 1-28. doi:10.1002/9781118539415.wbwell019

5. Bakker AB, Demerouti E. Job demands-resources theory: Taking stock and looking forward. J Occup Health Psychol. 2017;22(3):273-285. doi: 10.1037/ ocp0000056

6. Schaufeli WB, Bakker AB, Salanova M. The measurement of work engagement with a short questionnaire: a cross-national study. Educ Psychol Meas. 2006:;66(4):701-716. doi:10.1177/0013164405282471

7. Kulikowski K. Do we all agree on how to measure work engagement? Factorial validity of Utrecht Work Engagement Scale as a standard measurement tool - A literature review. Int J Occup Med Environ Health. 2017;30(2):161-175. doi: 10.13075/ijomeh.1896.00947.

8. Flores C, Fernández, M, Juárez A, Merino C, Guimet M. Entusiasmo por el trabajo (engagement): un estudio de validez en profesionales de la docencia en Lima, Perú. Liberabit. 2015;21(2):195-205. 
9. Maslach C, Jackson S, Leiter MP. Maslach Burnout Inventory Manual (3rd ed). Palo Alto, CA: Consulting Psychologist Press; 1996.

10. Fernández-Arata $M$, Juárez A, Merino C. Análisis estructural e invarianza de medición del MBI-GS en trabajadores peruanos. Liberabit. 2015;21(1):9-20.

11. Hanckock GR, Mueller RO. Rethinking construct reliability within latent variable systems. In Cudeck R, du Toit S, Sorbom D, Eds. Structural Equation Modeling: Present and Future-A Festschrift in Honor of Karl Joreskog. Lincolnwood: IL Scientific Software International; 2001. p. 195-216.

12. Marsh $\mathrm{H}, \mathrm{Hau}, \mathrm{K}$, Wen, Z. In search of golden rules: comment on hypothesis-testing approaches to setting cutoff values for fit indexes and dangers in overgeneralizing Hu and Bentler's (1999) findings. Struct Equ Modeling. 2004;11(3):320-341. doi: 10.1207/s15328007sem1103_2

13. McDonald RP. Test theory: A unified approach. Mahwah: Lawrence Erlbaum Associates; 1999.

14. Cronbach LJ. Coefficient alpha and the internal structure of tests. Psychometrika. 1951;16:297-334. doi: 10.1007/BF02310555

15. Kelley K, Pornprasertmanit P. Confidence intervals for population reliability coefficients: Evaluation of methods, recommendations, and software for homogeneous composite measures. Psychol Methods. 2016;26(1):69-92. doi: 10.1037/ a0040086

16. Yuan $\mathrm{KH}$. Zhang Z. Robust structural equation modeling with missing data and auxiliary variables. Psychometrika. 2012;77(4):803-826. doi: 10.1007/s11336-0129282-4

17. Gignac GE, Bates TC, Jang K. Implications relevant to CFA model misfit, reliability, and the Five Factor Model as measured by the NEO-FFI. Personality and Individual Differences. 2007;43(5):1051-1062. doi: 10.1016/j.paid.2007.02.024

18. Tong $X$, Zhang $Z$, Yuan $\mathrm{KH}$. Evaluation of test statistics for robust structural equation modeling with nonnormal missing data. Multivariate Behav Res. 2014;21(4):553-565. doi: 10.1080/10705511.2014.919820

19. Lee RT. Ashforth BE. A meta-analytic examination of the correlates of the three dimensions of job burnout. J Appl Psychol. 1996;81:123-133. doi: 10.1037/00219010.81.2.123 